\title{
Postoperative Hemorrhage, CTCAE
}

National Cancer Institute

\section{Source}

National Cancer Institute. Postoperative Hemorrhage, CTCAE. NCI Thesaurus. Code

C143772.

A disorder characterized by bleeding occurring after a surgical procedure. 